\title{
Speed Limit for Cell Growth
}

\author{
The composition of a cell's protein-synthesis machinery is tuned to \\ optimize the cell's reproduction rate.
}

\author{
By Stefan Klumpp
}

P eproduction is one of the characteristic features of all living things. Multicellular organisms, such as animals and humans, birth children that resemble their two parents, while individual cells divide to create a copy of themselves. At the molecular scale, reproduction occurs via molecular machines that synthesize the components of cells, as well as reproducing themselves. While researchers have analyzed the structure of these molecular machines in great detail, how that structure affects cell growth remains unclear. Now Sarah Kostinski and Shlomi Reuveni of Tel Aviv University, Israel, analyze the reproduction of a specific machine called a

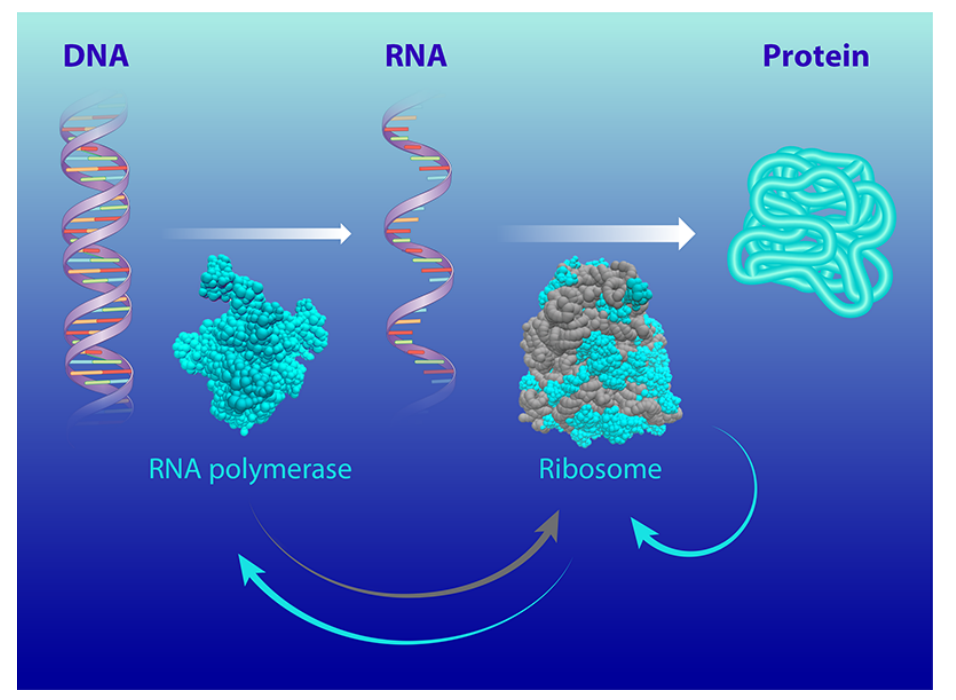

Figure 1: The molecular machines that process the genetic information from DNA and turn it into RNA and then into protein are themselves made of protein and RNA. A new analysis of the autocatalytic loops involved in this process (curved arrows) put constraints on how fast cells can reproduce [1].

Credit: APS/Carin Cain

ribosome, deriving from it a speed limit for the reproduction of cells [1]. They find that the speed depends on the composition of the ribosome, specifically the ratio of RNA to protein, with a maximal growth rate occurring for a 2:1 ratio. The result suggests that the ribosome's design is neither an accident of evolution nor a consequence of the ribosome's chemistry. Rather it is carefully tuned to maximize cell growth.

The ribosome is a large molecular complex that synthesizes proteins and that is found in all cells. To make proteins, a ribosome uses the cell's genetic information (transcribed from DNA to RNA) to join amino acids together in the correct order [2]. Bacterial ribosomes consist of around 50 individual proteins and three RNA molecules, which perform both structural and catalytic roles [2]. The larger RNA molecules make up about 2/3 of a ribosome's mass.

Ribosomes are closely linked with cell growth. In bacterial cultures, for example, the ribosome concentration has been found to depend on cell growth rate, increasing linearly up to some maximum growth rate $[3,4]$. This linear relation emerged from the quantitative characterization of the composition of bacterial cells $[5,6]$. It is called a growth law, in analogy to the phenomenological laws of physics, such as Ohm's law. The growth law provides a fruitful starting point for understanding how cells allocate their resources-in this case protein synthesis machinery-toward different functions $[3,7,8]$. But, despite over 60 years of work on this topic, researchers still don't fully understand what limits a cell's growth rate, a hole that the new work of Kostinski and Reuveni helps fill.

In their study, the duo connects this growth speed limit to the autocatalytic activity of the ribosomes. They develop a model that accounts for the activity of two molecular machines that 
are involved in cellular reproduction: the ribosome and the RNA polymerase (the molecular machine that synthesizes RNA).

The model considers how the molecular machines are themselves synthesized, a process that involves two autocatalytic loops (Fig. 1). The first loop accounts for the activity of the ribosomes, which synthesize all the bacteria's proteins, including the ribosomal proteins. The second loop accounts for the synthesis of ribosomal RNAs by RNA polymerases, which are made by ribosomes.

The team derives two speed limits for growth. The first, which has been known for a long time and relates to the first autocatalytic loop, states that a cell cannot duplicate faster than the time it takes one ribosome to make a complete set of ribosomal proteins for another ribosome. If a cell also contains other proteins (as all real cells do), duplicating those additional proteins adds to the so-called doubling time, the time needed to make a complete copy of the cell. Accounting for that leads to the known linear relation between cell growth rate (the inverse of the doubling time) and the observed ribosome concentration.

The second speed limit comes from factoring in the synthesis of the ribosomal RNA. Doing so, Kostinski and Reuveni find that the doubling time must also be larger than another synthesis time. This other time is the geometric mean of the time it takes a ribosome to make its share of RNA polymerases and the time it takes an RNA polymerase to make one copy of each ribosomal RNA.

Together, the two speed limits link a cell's growth to the composition of its ribosome: When the ribosome contains more protein, the first speed limit is lower. But the opposite is true for the second speed limit, which is higher when the ribosome contains more protein. Since the overall speed limit is the lower of the two, the RNA-to-protein composition that maximizes cell growth depends on the balance of these two limits. Using known values for the bacterium Escherichia coli, Kostinski and Reuveni fixed all other parameters in their two speed limit equations-such as the activity rates of the two molecular machines and sizes of the two molecules-finding an optimal RNA-to-protein ratio of approximately 2:1, as observed experimentally in bacterial ribosomes. Thus the result reinforces the idea that optimal design and optimal use of a cell's protein synthesis machinery are crucial for rapid cell growth.

So what's next? One avenue for further studies is looking at how evolution led to these speed limits. The composition of the ribosome is roughly uniform between all species (with the exception of the ribosomes in mitochondria). But the activity rates are expected to vary between species. If the 2:1 ratio is the result of an optimization by evolution, these parameters should have evolved together. This idea could be tested by characterizing how these parameters have evolved in different species, potentially providing additional limits on the design of the protein synthesis machinery.

This research is published in Physical Review Letters.

Stefan Klumpp: Institute for the Dynamics of Complex Systems, University of Göttingen, Göttingen, Germany

\section{REFERENCES}

1. Sarah Kostinski and Shlomi Reuveni, "Ribosome composition maximizes cellular growth rates in E. coli," Phys. Rev. Lett. 125, 028103 (2020).

2. D. L.J. Lafontaine and D. Tollervey, "The function and synthesis of ribosomes," Nat. Rev. Mol. Cell Biol. 2, 514 (2001).

3. M. Scott et al., "Interdependence of cell growth and gene expression: Origins and consequences," Science 330, 1099 (2010).

4. M. Scott et al., "Emergence of robust growth laws from optimal regulation of ribosome synthesis," Mol. Syst. Biol. 10, 747 (2014).

5. M. Schaechter et al., "Dependency on medium and temperature of cell size and chemical composition during balanced growth of Salmonella typhimurium,” J. Gen. Microbiol. 19, 592 (1958).

6. F. C. Neidhardt and B. Magasanik, "Studies on the role of ribonucleic acid in the growth of bacteria," Biochim. Biophys. Acta 42, 99 (1960).

7. D. Molenaar et al., "Shifts in growth strategies reflect tradeoffs in cellular economics," Mol. Syst. Biol. 5, 323 (2009).

8. O. Shoval et al., "Evolutionary trade-offs, pareto optimality, and the geometry of phenotype space," Science 336, 1157 (2012). 